\title{
Nef protein of human immunodeficiency virus type 1 binds its own myristoylated $\mathrm{N}$-terminus
}

\author{
Silke Hoffmann ${ }^{1,2}$, Esther Jonas ${ }^{1,2}$, Simone \\ König $^{3}$, Andrea Preusser-Kunze ${ }^{4, a}$ and Dieter \\ Willbold ${ }^{1,2, *}$ \\ ${ }^{1}$ Institut für Physikalische Biologie and BMFZ, Heinrich- \\ Heine-Universität, D-40225 Düsseldorf, Germany \\ ${ }^{2}$ Forschungszentrum Jülich, Institut für \\ Neurowissenschaften and Biophysik (INB-2), D-52425 \\ Jülich, Germany \\ ${ }^{3}$ Integrated Functional Genomics, Interdisciplinary \\ Clinical Research Center, Medical Faculty, University of \\ Münster, D-48149 Münster, Germany \\ ${ }^{4}$ Institut für Molekulare Biotechnologie, D-07745 Jena, \\ Germany \\ ${ }^{*}$ Corresponding author \\ e-mail: d.willbold@fz-juelich.de
}

\begin{abstract}
HIV-1 Nef is a small protein (approx. $25 \mathrm{kDa}$ ) that is posttranslationally modified by myristoylation. To explain its complex activities, a 'Nef-cycle' is discussed, which postulates different molecular conformations of Nef. Using recombinant full-length non-myristoylated Nef and synthetic peptides, we demonstrate by fluorescence titration experiments that a peptide representing the myristoylated N-terminus of Nef is specifically bound by Nef. A nonmyristoylated N-terminal fragment of Nef or a myristoylated control peptide does not bind to Nef. These results are the first direct experimental evidence of the existence of a myristate-binding pocket in Nef, a prerequisite of the postulated 'closed' Nef conformation.
\end{abstract}

Keywords: fluorescence titration; peptide binding; protein conformation.

Human immunodeficiency virus type 1 (HIV-1) Nef is a small protein of approximately $25 \mathrm{kDa}$ that is essential for high-titer viral replication and pathogenesis of acquired immunodeficiency syndrome (AIDS). Nef is produced in the earliest stage of viral gene expression and is a component of viral particles. It is posttranslationally modified by myristoylation of the $\mathrm{N}$-terminal glycine (residue 2 in the SwissProt numbering scheme) and by phosphorylation of at least one serine residue. Nef interacts with a multitude of host cellular proteins. Its multiple functions, e.g., its interaction with various signal transduction proteins such as p21-activated kinase and cytoplasmic protein tyrosine kinases (Renkema and Saksela, 2000)

a Present address: Georg-August-Universität Göttingen, Abteilung Biochemie II, D-37073 Göttingen, Germany. and Nef-mediated down-modulation of CD4, MHC class I, CD28, CXR4 and CD8 $\alpha \beta$ (Mangasarian et al., 1999; Bell et al., 2001; Swigut et al., 2001; Hrecka et al., 2005; Stove et al., 2005), involve cytoplasmic and membranebound stages. The loss of a special Nef function, namely the Nef-mediated suppression of T-cell activation, is held to be responsible for the progression to AIDS in HIV-1infected humans, which is contrary to the non-pathogenic processing of most SIV infections (Schindler et al., 2006). Very recently, interference in the maturation of stimulatory T-cell contacts by modulation of N-WASP activity through Nef was described (Haller et al., 2006). To explain the complex activities of the viral protein, a 'Nef-cycle' is discussed, which postulates different molecular conformations of Nef (Arold and Baur, 2001; Dennis et al., 2005). It is hypothesized that Nef undergoes a substantial conformational change from an 'open' to a 'closed' form, whereby the myristate group is thought to be sequestered into a binding pocket. Myristoylated Nef may then switch to the open conformation by association of the $\mathrm{N}$-terminal region with membranes. Experimental proof of such a myristate-binding pocket in Nef, however, is still lacking. Structural studies by nuclear magnetic resonance (NMR) spectroscopy or X-ray crystallography to address the postulated binding pocket are severely hampered by the extremely high aggregation tendency of myristoylated Nef (myr-Nef) at concentrations necessary for these experiments (Dennis et al., 2005). Therefore, we used another approach to investigate the potential myristate-binding pocket of Nef. Although it was reported that myristate is not bound by Nef (Geyer et al., 2001), it may well be that myristate is bound by Nef if covalently linked to the $\mathrm{N}$-terminal part of Nef. To address this question, we used unmodified recombinant full-length Nef protein starting with residue glycine $2\left(\mathrm{Nef}_{2-210}\right)$ and studied its binding to a synthetic

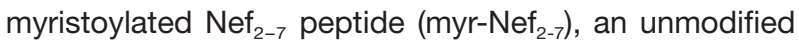
$\mathrm{Nef}_{2-7}$ peptide, and a myristoylated control peptide (myrPep) (Figure 1).

$\mathrm{Nef}_{2-210}$ was expressed in E. coli BL21(DE3) RIL cells harboring plasmid pUbi-Nef ${ }_{2-210}$ carrying the Nef gene

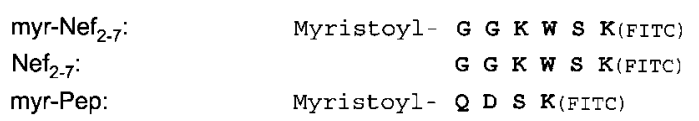

Figure 1 Overview of the FITC peptides used for Nef binding studies.

Amino acid sequences are given using the one letter-code for the peptides named on the left. The myristoyl modification, as well as the position of the fluorescein isothiocyanate label (FITC), is indicated. All peptides were purchased from JPT Peptide Technologies GmbH (Berlin, Germany), with their C-termini blocked by amidation. 
from the HIV-1 isolate SF2 (SwissProt accession no. $\mathrm{P} 03407)$ as a poly-histidine-tagged (His-tagged), ubiquitin-fused construct in a pTKK19xb/ub vector (Kohno et al., 1998). Bacteria were grown aerobically at $37^{\circ} \mathrm{C}$ in Luria broth containing kanamycin $(100 \mu \mathrm{g} / \mathrm{ml})$ and chloramphenicol $(34 \mu \mathrm{g} / \mathrm{ml})$. Cells at $\mathrm{OD}_{600 \mathrm{~nm}}$ of $\sim 0.8$ were induced with isopropyl $\beta$-D-thiogalactopyranoside (IPTG; final concentration $0.2 \mathrm{mM}$ ) for $3 \mathrm{~h}$, harvested by centrifugation and sonified in lysis buffer $\left(50 \mathrm{~mm} \mathrm{NaH_{2 }} \mathrm{PO}_{4}\right.$, $500 \mathrm{~mm} \mathrm{NaCl}, 10 \mathrm{~mm}$ imidazole, $\mathrm{pH}$ 8.0, supplemented with lysozyme and protease inhibitors). The extract was clarified by centrifugation and applied to a Ni-NTA metal affinity column (Qiagen, Hilden, Germany). After extensive washing $\left(50 \mathrm{~mm} \mathrm{NaH}{ }_{2} \mathrm{PO}_{4}, 500 \mathrm{~mm} \mathrm{NaCl}, 10 \mathrm{~mm}\right.$ imidazole), the protein was eluted by increasing the imidazole concentration stepwise from 10 to $250 \mathrm{~mm}$. Fractions containing pure fusion protein (usually fractions with $100 \mathrm{~mm}$ imidazole), as determined by denaturing SDSPAGE, were pooled and dialyzed against cleavage buffer and subsequently cleaved using His-tagged yeast ubiquitin hydrolase (Kohno et al., 1998). $\mathrm{Nef}_{2-210}$ was separated from His-tagged ubiquitin, uncleaved fusion protein and His-tagged yeast ubiquitin hydrolase by a second $\mathrm{Ni}$ NTA metal affinity column equilibrated with cleavage buffer, with $\mathrm{Nef}_{2-210}$, notably without any artificial additional amino acid residue, located in the flow-through (Figure 2). To assure protein solubility, fractions containing pure $\mathrm{Nef}_{2-210}$ were directly supplemented with $\mathrm{NaCl}$ to a final concentration of $300 \mathrm{~mm}$ and concentrated using an Amicon flow cell under $\mathrm{N}_{2}$ atmosphere. All buffers used in this study were degassed and supplemented with $14 \mathrm{~mm} \beta$-mercaptoethanol. The identity of the $\mathrm{Nef}_{2-210}$ protein was confirmed by in-gel tryptic digestion of the electrophoretically separated protein and mass spectrometric peptide mapping. The final yield of $\mathrm{Nef}_{2-210}$ was $8 \mathrm{mg} / \mathrm{l}$.

Fluorescence titrations were carried out by measuring the fluorescence of a peptide comprising residues 2-7 of Nef (GGKWSK), with the N-terminal glycine being myristoylated and the $\mathrm{C}$-terminal lysine carrying a fluo-

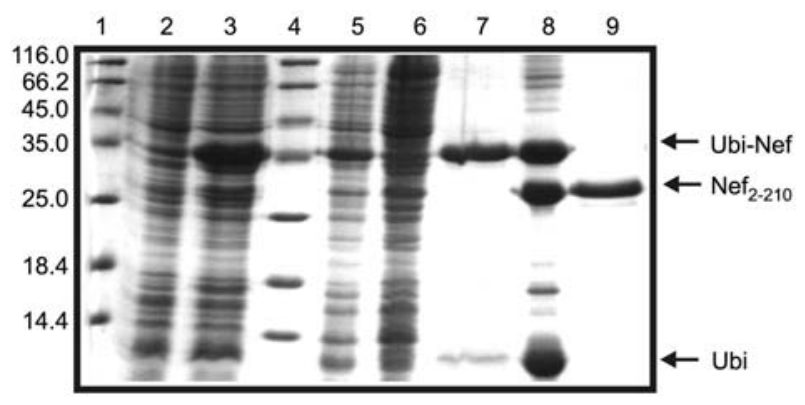

Figure 2 Expression and purification of recombinant $\mathrm{Nef}_{2-210}$ from HIV-1 isolate SF2.

Expression of pUbi-Nef in E. coli (DE3) RIL before (lane 1) and after (lane 2) induction with $0.2 \mathrm{mM}$ IPTG. Purification of $\mathrm{Nef}_{2-210}$ : soluble fraction of the cell lysate (lane 5); flow-through fraction (lane 6) and pooled His-tagged ubiquitin-Nef fusion protein (UbiNef) containing fractions (lane 7) after $\mathrm{Ni}^{2+}$-chelating affinity chromatography; cleavage (lane 8) of Ubi-Nef with yeast ubiquitin hydrolase and second $\mathrm{Ni}^{2+}$-chelating affinity chromatography (lane 9); $\mathrm{Nef}_{2-210}$ is located in the flow-through fraction; $\mathrm{M}$, molecular mass markers (in kDa). Ubi-Nef, Nef(2-210) and Histagged ubiquitin (Ubi) are marked by arrows. rescein isothiocyanate (FITC) residue covalently linked to

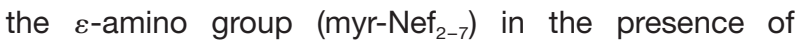
increasing concentrations of $\mathrm{Nef}_{2-210}$ (Figure 3A). Titrations were performed as previously described, and no binding of Nef to the FITC label was found (Preusser et al., 2001). Assuming a simple bimolecular reaction between Nef and the peptides, analysis by non-linear curve fitting yielded a $K_{d}$ value of $8.7 \mu \mathrm{M}$ with a standard deviation of 2.0. No binding was observed for the unmyristoylated $\mathrm{Nef}_{2-7}$ peptide (Figure 3A). As a further control, an arbitrarily selected myristoylated control peptide (myrPep) was also titrated with Nef (Figure 3A). Owing to the extremely weak binding indicated by the linear decrease in Nef-concentration-dependent fluorescence, a dissociation constant could not be determined, but is clearly greater than $50 \mu \mathrm{M}$. Linear regression analysis of the Scatchard plot of myr- $\mathrm{Nef}_{2-7}$ titration with Nef confirmed the dissociation constant $\left(-1 / K_{d}\right.$, slope). In addition, the $\mathrm{x}$-axis intercept of the Scatchard plot yielded the number of Nef binding sites per myr-Nef ${ }_{2-7}$ peptide (Figure 3B). The value is slightly greater than 1 , probably due to partial oligomerization of Nef leading to a lower apparent concentration of Nef monomers. Such a concentrationdependent oligomerization of non-myristoylated Nef is well known and is fully reversible (Arold et al., 2000).
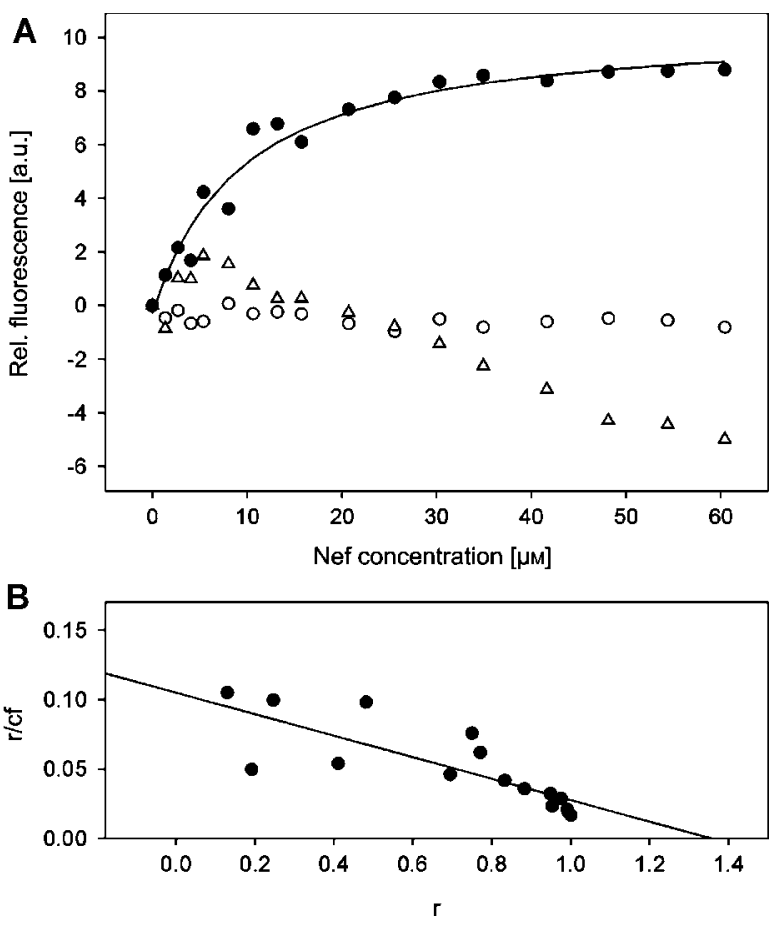

Figure 3 Fluorescence titration studies.

(A) Fluorescence of $1.0 \mu \mathrm{M}$ myr-Nef $f_{2-7}(\bullet), 1.0 \mu \mathrm{M} \mathrm{Nef} f_{2-7}(\bigcirc)$ and $1.0 \mu \mathrm{M}$ control peptide (myr-Pep) $(\Delta)$ as a function of Nef concentration. Each titration was repeated twice and yielded fully reproducible results. The non-linear fit obtained for Nef binding to myr-Nef ${ }_{2-7}$ is shown by a solid line. (B) Scatchard plot of the data points shown in (A) for binding of HIV-1 Nef to myr-Nef ${ }_{2-7}$ $(\bullet)$ peptide. The $x$-axis $(r)$ corresponds to the ratio of bound Nef to total myr- $\mathrm{Nef}_{2-7}$ peptide concentration, and $\mathrm{r} / \mathrm{cf}$ on the $\mathrm{y}$-axis is the ratio of $r$ and unbound Nef. Linear regression analysis (solid line) yielded the dissociation constant $\left(-1 / K_{d}\right.$, slope), and the number of Nef binding sites per myr-Nef ${ }_{2-7}$ peptide molecule (x-axis intercept). 
The titration experiments in our study clearly show that full-length Nef is able to bind a peptide comprising its own $\mathrm{N}$-terminus, if and only if it is $\mathrm{N}$-terminally myristoylated. This is the first direct experimental evidence of the existence of such a binding site on Nef. This in turn is a prerequisite for the postulated 'closed' form of Nef, which is defined by an interaction between the myristoylated $\mathrm{N}$-terminus and the core. The closed conformation of Nef helps to explain why modified Nef is approximately $50 \%$ soluble when being expressed recombinantly (Dennis et al., 2005), and remains cytosolic in HeLa cells to approximately $75 \%$ (Bentham et al., 2006). The binding site of Nef for its myristoylated N-terminus nicely explains the drastically increased aggregation tendency of myristoylated Nef compared to unmodified Nef at higher protein concentrations. Sequestration of the myristoylated $\mathrm{N}$-terminus by intramolecular contacts has been suggested as the mechanism that renders modified Nef soluble and monomeric at low protein concentrations (Dennis et al., 2005). We assume that at higher protein concentrations, at least in vitro, intermolecular interactions between myristoylated Nef molecules may occur, potentially leading to high-molecular-weight assemblies. Beside the 'open' conformation of modified Nef discussed, in which largely hydrophobic surfaces are exposed, this might result in insolubility and aggregation, and might be one reason for the many different Nef species described, e.g., during dynamic light scattering experiments (Dennis et al., 2005).

It is hypothesized that contact with the cellular membrane could trigger a conformational change in Nef, thus relieving the interaction between the myristoylated $\mathrm{N}$-terminus and the core, which subsequently might expose various interaction motifs of Nef to other factors. This regulatory step might be an interesting target for novel therapeutic approaches to HIV.

\section{Acknowledgments}

We thank T. Kohno, Mitsubishi Kasei Institute of Life Sciences, Tokyo, Japan for providing the expression plasmid for poly-histidine-tagged yeast ubiquitin hydrolase and plasmid pTKK19xb/ $\mathrm{ub}$ as the basis for construction of the plasmid pUbi-Nef. This work was supported by a grant from Deutsche Forschungsgemeinschaft to D.W. (Wi 1472/4-2).

\section{References}

Arold, S., Hoh, F., Domergue, S., Birck, C., Delsuc, M.A., Jullien, M., and Dumas, C. (2000). Characterization and molecular basis of the oligomeric structure of HIV-1 nef protein. Protein Sci. 9, 1137-1148.
Arold, S.T. and Baur, A.S. (2001). Dynamic Nef and Nef dynamics: how structure could explain the complex activities of this small HIV protein. Trends Biochem. Sci. 26, 356-363.

Bell, I., Schaefer, T.M., Trible, R.P., Amedee, A., and Reinhart, T.A. (2001). Down-modulation of the costimulatory molecule, CD28, is a conserved activity of multiple SIV Nefs and is dependent on histidine 196 of Nef. Virology 283, 148-158.

Bentham, M., Mazaleyrat, S., and Harris, M. (2006). Role of myristoylation and $\mathrm{N}$-terminal basic residues in membrane association of the human immunodeficiency virus type $1 \mathrm{Nef}$ protein. J. Gen. Virol. 87, 563-571.

Dennis, C.A., Baron, A., Grossmann, J.G., Mazaleyrat, S., Harris, M., and Jaeger, J. (2005). Co-translational myristoylation alters the quaternary structure of HIV-1 Nef in solution. Proteins 60, 658-669.

Geyer, M., Fackler, O.T., and Peterlin, B.M. (2001). Structurefunction relationships in HIV-1 Nef. EMBO Rep. 2, 580-585.

Haller, C., Rauch, S., Michel, N., Hannemann, S., Lehmann, M.J., Keppler, O.T., and Fackler, O.T. (2006). The HIV-1 pathogenicity factor Nef interferes with maturation of stimulatory Tlymphocyte contacts by modulation of N-Wasp activity. J. Biol. Chem. 281, 19618-19630.

Hrecka, K., Swigut, T., Schindler, M., Kirchhoff, F., and Skowronski, J. (2005). Nef proteins from diverse groups of primate lentiviruses downmodulate CXCR4 to inhibit migration to the chemokine stromal derived factor 1. J. Virol. 79, 1065010659.

Kohno, T., Kusunoki, H., Sato, K., and Wakamatsu, K. (1998). A new general method for the biosynthesis of stable isotopeenriched peptides using a decahistidine-tagged ubiquitin fusion system: an application to the production of mastoparan-X uniformly enriched with ${ }^{15} \mathrm{~N}$ and ${ }^{15} \mathrm{~N} /{ }^{13} \mathrm{C}$. J. Biomol. NMR 12, 109-121.

Mangasarian, A., Piguet, V., Wang, J.K., Chen, Y.L., and Trono, D. (1999). Nef-induced CD4 and major histocompatibility complex class I (MHC-I) down-regulation are governed by distinct determinants: $\mathrm{N}$-terminal $\alpha$-helix and proline repeat of Nef selectively regulate MHC-I trafficking. J. Virol. 73, 1964-1973.

Preusser, A., Briese, L., Baur, A.S., and Willbold, D. (2001). Direct in vitro binding of full-length human immunodeficiency virus type 1 Nef protein to CD4 cytoplasmic domain. J. Virol. 75, 3960-3964.

Renkema, G.H. and Saksela, K. (2000). Interactions of HIV-1 NEF with cellular signal transducing proteins. Front. Biosci. 5, D268-D283.

Schindler, M., Munch, J., Kutsch, O., Li, H., Santiago, M.L., Bibollet-Ruche, F., Muller-Trutwin, M.C., Novembre, F.J., Peeters, M., Courgnaud, V., et al. (2006). Nef-mediated suppression of $T$ cell activation was lost in a lentiviral lineage that gave rise to HIV-1. Cell 125, 1055-1067.

Stove, V., Van de Walle, I., Naessens, E., Coene, E., Stove, C., Plum, J., and Verhasselt, B. (2005). Human immunodeficiency virus Nef induces rapid internalization of the T-cell coreceptor CD8 $\alpha \beta$. J. Virol. 79, 11422-11433.

Swigut, T., Shohdy, N., and Skowronski, J. (2001). Mechanism for down-regulation of CD28 by Nef. EMBO J. 20, 15931604.

Received July 28, 2006; accepted September 1, 2006 